\title{
Evaluation of Crisis, Reversibility, Alert Management for Constrained Dynamical Systems Using Impulse Dynamical Systems
}

\author{
Patrick Saint-Pierre \\ Centre de Recherche Viabilité, Jeux, Contrôle \\ Université Paris IX - Dauphine \\ saint-pierre@viab.dauphine.fr
}

\begin{abstract}
Considering constrained dynamical systems characterized by a differential inclusion $x^{\prime} \in F(x)$, we are interested in studying the situation where, for various reasons, the state leaves the constrain domain $K$ either because the initial position does not belong to the Viability Kernel of $K$ for $F$ or it belongs to a "sustainable or tolerable" but not "comfortable" domain. This question appears in numerous models in Social Sciences or in Genetics as well as for controlling security in Automatics and Robotics, like Aircraft landing, rolling and taking off. After recalling basic concepts in Viability Theory and using hybrid calculus, we show how to evaluate and manage crisis in general cases.
\end{abstract}

\section{Introduction}

We consider a dynamical impulse system describing the evolution of a state variable $x \in X$ which should remain in a constrained domain. Research efforts are devoted to the study of such systems mainly in extending the mathematical tools of the Control Theory ([5, Bensoussan \& Menaldi] in order to take into account as well constraints (qualitative properties) as optimality performance (quantitative properties), and in developing methods for non linear hybrid systems (see for instance [19, Sastry], [2, Aubin]), [4, Tomlin, Crück, Bayen, and ref.]).

On the other hand the study of controlled system with state constraints has been widely developed this last decade using the main concepts of SetValued Analysis [3, Aubin \& Frankowska] and Numerical Set-Valued Analysis [16, Saint-Pierre] which can be regarded as a "Tool Box" for viability theory and numerical viability (viability, equilibria, stability, reachability, controllability, optimal control (minimal time-to-reach problem, infinite horizon optimal control [6, Cardaliaguet, Quincampoix \& Saint-Pierre], differential games (discriminating and leadership kernels, conditional and guaranteed strategies, minimal hitting time function, robustness, qualitative analysis [13, Leitmann], [18, Seube], [9, Dordan] and their applications to mathematical economics, finance, demography or biology as well as to enginery and automatics when taking into account constraints is unescapable.

Crisis time function has been introduced in [10, Doyen \& Saint-Pierre]), allowing the characterization of reversibility and irreversibility of the lapse for constraints. In particular these concepts can be found for instance in Environmental 
Protection models (preservation of future generation, precaution principle) or in Biodiversity (maintaining polymorphism).

The Viability Kernel Algorithm originally designed for computing viability kernels in the frame of set-valued analysis has been widen to approximate the smallest lower semicontinuous subsolution of the Hamilton-Jacobi-Belmann equation [11, Frankowska], [6, Cardaliaguet, Quincampoix \& Saint-Pierre]. Moreover viability techniques have been extended to hybrid systems first for computing the Hybrid Kernel which broadens the concept of Viability Kernel, second for computing the Minimal Cost function in non smooth contexts and in the presence of uncertainty [8, Crück]. The scope of this paper is to show how Viability Theory applied in the context of hybrid systems allows to manage crisis that is to say situations where precisely viability fails.

\section{Continuous, Discrete and Hybrid Systems}

\subsection{Definitions}

Let $x$ denote the state vector belonging to a finite dimensional vector space $X$.

- A continuous evolution is given by a set-valued map $F: X \rightsquigarrow X$ with convex compact values, closed graph defined on a closed set $D \subset X$.

$$
x^{\prime}(t) \in F(x(t)) \text {, for almost all } t \in \mathbb{R}^{+} .
$$

For instance, $F(x)=\{f(x, u)$, where $u \in U(x)\}$.

- A discrete (or impulsive) evolution is given by a set-valued map $\Phi$ with compact values, closed graph defined on a closed set $C \subset X$.

$$
x^{n+1} \in \Phi\left(x^{n}\right) .
$$

A dynamical system is said to be continuous if $C=\emptyset$, discrete if $D=\emptyset$ and hybrid if $C \cap D \neq \emptyset$.

We denote $\mathcal{S}_{F}^{c}(x)$ the set of all absolutely continuous solutions to the differential inclusion (1) with initial condition $x(0)=x$ and by $\mathcal{S}_{\Phi}^{d}(x)$ the set of all sequences solutions to the recursive inclusion (2) with initial condition $x^{0}=x$.

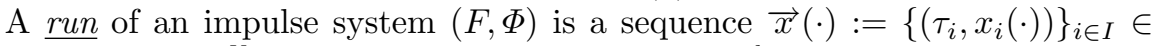
$\left(\mathbb{R}^{+} \times \overline{\mathcal{C}}(0, \infty ; X)\right)^{\mathbb{N}}$, where $x_{i}(\cdot) \in \mathcal{S}_{F}^{c}\left(x_{i}(0)\right)$ is the $i^{\text {th }}$ motive which is an absolutely continuous solution to (1) starting from $x_{i}=x_{i}(0)$ at time 0 until time $\tau_{i}$ and $x_{i+1} \in \Phi\left(x_{i}\left(\tau_{i}\right)\right)$.

We denote $\mathcal{S}_{F, \Phi}^{h}(x)$ the set of all runs associated with both differential and recursive inclusions with initial condition $x_{0}=x$.

\section{Viability Kernels}

Let $D \subset X$. We denote $\mathcal{D}$ the set of all functions defined on $R$ with values in $D$ and $\overrightarrow{\mathcal{D}}$ the set of all sequences contained in $D$. Let us recall the following definitions and properties.

A closed set $D$ is a viability domain for $F$ if and only if $\forall x \in D, S_{F}^{c}(x) \cap \mathcal{D} \neq \emptyset$ (Qualitative Property). This is equivalent to the following properties:

i) - $\forall x \in D, F(x) \cap T_{D}(x) \neq \emptyset$ (Geometrical Property)

ii) - $\forall x \in D, \forall p \in N P_{D}(x), H(x, p) \leq 0$ (Dual Property) 
where $T_{D}(x)$ is the contingent cone to $D$ at $x, N P_{D}(x)$ is the set of proximal normal to $D$ at $x$ and $H(x, p):=\min _{y \in F(x)}<y, p>$ is the associated Hamiltonian. The largest closed viability domain contained in a closed set $K \subset X$ exists ([1]). It is called the Viability Kernel of $K$ for $F$ and is denoted $\operatorname{Viab}_{F}(K)$.

A set $D$ is a discrete viability domain for $\Phi$ if and only if $\forall x \in D, S_{\Phi}^{d}(x) \cap \vec{D} \neq$ $\emptyset$ (Qualitative Property). This is equivalent to the following property:

$\forall x \in D, \Phi(x) \cap D \neq \emptyset$ (Geometrical Property)

The largest closed discrete viability domain contained in $K$ exists $([16])$. It is called the Discrete Viability Kernel of $K$ for $\Phi$ and is denoted $\overrightarrow{V i a b}_{\Phi}(K)$.

\subsection{Impulse systems with state constraints and Hybrid Viability Kernels}

Let $\vec{x}(\cdot):=\left\{\left(\tau_{i}, x_{i}(\cdot)\right)\right\}_{i \in I} \in\left(\mathbb{R}^{+} \times X \times \mathcal{C}(0, \infty ; X)\right)^{\mathbb{N}}$ be a run associated with the hybrid dynamical system $(1,2)$. We set $T=\sum_{i=0}^{n} \tau_{i}$.

Let be $\delta>0$ and consider sequences $\left(t_{i}\right)_{i \in I}$ and $\left(\vartheta_{i}\right)_{i \in I}$ given by $t_{0}=\vartheta_{0}=$ $0, \quad t_{i+1}=t_{i}+\tau_{i}$ and $\vartheta_{i+1}=\vartheta_{i}+\tau_{i}+\delta$. We have $\vartheta_{i+1}=t_{i}+i \delta$. With any $t \in[0, T]$ we associate $i_{t}$ satisfying $t_{i_{t}} \leq t<t_{i_{t}+1}$, with any $\vartheta \in \mathbb{R}$ we associate $i_{\vartheta}$ satisfying $\vartheta_{i_{\vartheta}} \leq \vartheta<\vartheta_{i_{\vartheta}+1}$. and with a run $\vec{x}(\cdot)$ we associate

$$
x^{e}(\vartheta)=x_{0}+\sum_{i=0}^{i_{\vartheta}}\left(\int_{0}^{\tau_{i}^{\star}} x_{i}^{\prime}(\tau) d \tau+\alpha_{i}^{\star}\left(x_{i+1}-x_{i}\left(\tau_{i}\right)\right)\right)
$$

where the motive $x_{i}(\tau)$ starts from $x_{i}=x_{i}(0)$ at time 0 until time $\tau_{i}, \tau_{i}^{\star}=$ $\min \left(\tau_{i}, \vartheta-\vartheta_{i}\right)$ and $\alpha_{i}^{\star}=\max \left(0, \min \left(1, \vartheta-\vartheta_{i}-\tau_{i}\right)\right)$.

We call hybrid solution associated with a run $\vec{x}(\cdot)$ the map $t \rightarrow x(t)$ given by $\left.x(t)=x^{\bar{e}\left(t+i_{t}\right.} \delta\right)$.

Definition - The hybrid viability kernel of $K$ for the impulse system $(F, \Phi, K)$ is the largest closed subset of initial states belonging to $K$ from which starts at least one hybrid viable solution. We denote this set $H y b_{(F, \Phi)}(K)$.

The Hybrid Viability Kernel can be approximated by a sequence of discrete viability kernels associated with suitable discrete systems [17, Saint-Pierrre].

\subsection{Assumptions and Approximated Impulse Systems}

- $C$ is compact and $\Phi: C \rightsquigarrow X$ is upper semi-continuous compact valued $0<\delta_{\text {inf }} \leq \inf _{x \in C} \inf _{y \in \Phi(x)}\|x-y\| \leq \sup _{x \in C} \sup _{y \in \Phi(x)}\|x-y\| \leq \delta_{\text {sup }}$ so that $\Phi$ has no fix point and its graph is closed. We set $\forall x \notin C, \Phi(x)=\emptyset$.

- $K$ is a compact set and $F$ is a Marchaud map satisfying $\sup _{x \in K} \sup _{y \in F(x)}\|y\| \leq M$. This assumption implies that $F$ is closed. As usual in the context of set-valued numerical analysis we assume that a "good" approximation $F_{\rho}$ of $F$ exists in the sense that $\operatorname{Graph}\left(F_{\rho}\right)$ remains in a not too large neighborhood of $F[16$, Saint-Pierrre]. 


\section{Management of Crisis}

\subsection{Reversibility and irreversibility}

The notion of Crisis corresponds to the situation where the evolution of the state described by a differential inclusion violates the constraints.

Viability Theory discusses conditions allowing the state maintained in the constraint set. However important questions arise:

1 - What to say and to do when the state leaves the constraint set?

2 - Can we predict for a given initial position a future crisis ?

3 - If a crisis occurs or if it is unavoidable in the future, what to say about its reversibility, its duration and how to recover Viability?

4 - Is it possible to define a scale of viability measuring seriousness of crisis to come and to numerically evaluate crisis ?

Consider a dynamical system $x^{\prime} \in F(x)$ and two closed subsets $K^{s} \subset K^{h} \subset$ $X$ representing "soft" constraints (viable, tolerable, comfortable,...) and "hard" constraints (unsustainable, unbearable, fatal,...).

\subsection{The Reversibility Domain}

We look for the set of initial position in $K_{h}$ from which there exists at least one solution which, if it leaves for a while the constraint set $K_{s}$, remains in $K_{h} \backslash K_{s}$ during a finite laps of time before coming back and forever remaining in $K_{s}$. We denote this set $\operatorname{Rever}_{F}\left(K_{h} ; K_{s}\right)$.

The set $\operatorname{Rever}_{F}\left(K_{h} ; K_{s}\right)$ is the capture domain of target $\left.\operatorname{Viab}_{F}\left(K_{s}\right)\right)$ for $F$ under constraint $K_{h}([15])$.

Indeed any solution which comes back and definitively remains in $K_{s}$ necessarily comes back in the Viability Kernel of $K_{s}$.

Moreover, if $\operatorname{Viab}_{F}\left(K_{h}\right)=\operatorname{Viab}_{F}\left(K_{s}\right), \operatorname{Rever}_{F}\left(K_{h} ; K_{s}\right)$ is the Viability Kernel of $K_{h}$ with target $\operatorname{Viab}_{F}\left(K_{s}\right)$ [15, Quincampoix \& Veliov].

\subsection{The Minimal Crisis Time function}

Consider the map which evaluates the minimal time the state will spend out of $K^{s}$ while remaining in $K^{h}$ when starting from an initial position $x$ :

$$
\begin{aligned}
& C_{K^{s}, F}^{K^{h}}(x)=\inf _{\left.x(\cdot) \in \mathcal{S}_{F}(x)\right) \cap \mathcal{K}^{h}} \mu\left(t \mid x(t) \notin K^{s}\right) \\
& =\inf _{x(\cdot) \in \mathcal{S}_{F}^{c}(x) \cap \mathcal{K}^{h}} \int_{0}^{+\infty} \mathcal{X}_{X \backslash K^{s}}(x(s))\left(1+\mathcal{I}_{\mathcal{K}^{h}}(x(s))\right) d s,
\end{aligned}
$$

where $\mu$ denotes the Lebesgue measure in $\mathbb{R}, \mathcal{X}$ and $\mathcal{I}$ stand for the characteristic and the indicatrix functions. We impose that $C_{K^{s}, F}^{K^{h}}(x)=+\infty$ whenever every trajectory $x(\cdot)$ starting from $x$ violates the constraints $K^{s}$ during an infinite time.

Theorem 1 [Doyen \& Saint-Pierre] If $F: X \rightsquigarrow X$ is a Marchaud set-valued map, then

a) $\forall x \in \operatorname{Dom}\left(C_{K^{s}, F}^{K^{h}}\right)$, the infimum is reached: $\exists x^{*}(\cdot) \in \mathcal{S}_{F}^{K^{h}}(x)$ such that

$$
C_{K^{s}, F}^{K^{h}}(x)=\int_{0}^{+\infty} \mathcal{X}_{X \backslash K^{s}}\left(x^{*}(s)\right)\left(1+\mathcal{I}_{\mathcal{K}^{h}}\left(x^{*}(s)\right)\right) d s,
$$


b) We have $\operatorname{Epi}\left(C_{K^{s}, F}^{K^{h}}\right)=\operatorname{Viab}_{\widetilde{F}}\left(K^{h} \times \mathbb{R}^{+}\right)$, where $\widetilde{F}$ corresponds to the extended dynamic with closed convex righthand side

$$
\left\{\begin{array}{l}
x^{\prime} \in F(x) \\
\left.y^{\prime} \in-\overline{c o\left(\mathcal{X}_{X \backslash K^{s}}\right)}(x)\right),
\end{array}\right.
$$

c) $C_{K^{s}, F}^{K^{h}}(\cdot)=\vartheta_{\operatorname{Viab}_{F}\left(K^{s}\right)}^{K^{h}}(\cdot)$ : the crisis map is lower semi-continuous and coincides with the Minimal Time-to-reach function where the constraint is $K^{h}$ and the target is $\operatorname{Viab}_{F}\left(K^{s}\right)$.

d) $\bullet \operatorname{Viab}_{F}\left(K^{s}\right)$ is the 0-level set of $C_{K^{s}, F}^{K^{h}}:\left\{x \in K^{h} \mid C_{K^{s}, F}^{K^{h}}(x)=0\right\}$.

Proof -

a) Existence of the infimum derives from an Aubin's theorem which states that the graph of the restriction of $\mathcal{S}_{F}(\cdot)$ to any compact is compact and from Fatou's Lemma.

b) Marchaud property of the set-valued map $\mathcal{X}_{K^{s}}^{\natural}(\cdot)$ implies existence of a Viability Kernel. Then we prove that $\operatorname{Epi}\left(C_{K^{s}, F}^{K^{h}}\right) \subset \operatorname{Viab}_{\widetilde{F}}\left(K^{h} \times \mathbb{R}^{+}\right)$.

Conversely we prove that for any $(x, y) \in \operatorname{Viab}_{\widetilde{F}}\left(K^{h} \times \mathbb{R}^{+}\right)$there exists a solution $x(\cdot)$ such that $\int_{0}^{\infty} \mathcal{X}_{X \backslash K^{s}}(x(s)) d s \leq y$ and deduce that $C_{K^{s}, F}^{K^{h}}(x)=$ $\inf _{x(\cdot) \in \mathcal{S}_{F}^{c}(x) \cap \mathcal{K}^{h}} \int_{0}^{+\infty} \mathcal{X}_{X \backslash K^{s}}(x(t)) d t \leq y$. Therefore $\operatorname{Viab}_{\widetilde{F}}\left(K^{h} \times \mathbb{R}^{+}\right) \subset \operatorname{Epi}\left(C_{K^{s}, F}^{K^{h}}\right)$.

\subsection{Approximation Algorithm}

From Theorem 1, the Viability Algorithm provides an algorithm to approximate $C_{K^{s}, F}^{K^{h}}(\cdot)$. Let $\rho>\tau>0$ be the time and the space steps such that $\frac{\tau}{\rho} \rightarrow 0^{+}$.

Let $X_{\tau}$ be a grid of $X$ with mesh of size $\tau$ and $K_{\tau}^{h}:=\left(K^{h}+\tau \mathcal{B}\right) \cap X_{\tau}$.

We set $\mathcal{K}_{\tau}^{h, 0}=\mathcal{K}_{\tau}^{h}:=K_{\tau}^{h} \times \tau \mathbb{Z}^{+}$and $C_{\tau}^{0}: K_{\tau}^{h} \rightarrow \tau \mathbb{Z}^{+}$, the lower semicontinuous function defined by $\operatorname{Epi}\left(C_{\tau}^{0}\right)=\mathcal{K}_{\tau}^{h, 0}$.

With the sequence $\mathcal{H}_{\tau}^{h, n}$ defined by the Viability Kernel Algorithm ([16]):

$$
\mathcal{H}_{\tau}^{h, n+1}:=\mathcal{H}_{\tau}^{h, n} \cap\left(\mathcal{I}+\tau \tilde{F}_{\tau}\right)^{-1}\left(\mathcal{H}_{\tau}^{h, n}\right)
$$

we associate the functions $C_{\tau}^{n}: K_{\tau}^{h} \rightarrow \tau \mathbb{Z}$ which epigraph coincides with $\mathcal{H}_{\tau}^{h, n}$.

Proposition 2 Functions $C_{\tau}^{n}$ are lower semicontinuous and satisfy:

$C_{\tau}^{n+1}(x):=\left\{\begin{array}{ll}\left.\rho-\tau+\min _{v \in F(x) b \in \mathcal{B}} C_{\tau}^{n}(x+\rho(v+\phi(\rho)) b)\right), & \text { if } x \in K^{h}, d_{C}(x)>M \rho+\tau \\ C_{\tau}^{n}(x) & \text { if not, }\end{array}\right.$.

The limit exists $\forall x \in K_{\tau}^{h}: C_{\tau}^{\infty}(x):=\lim _{n \rightarrow+\infty} C_{\tau}^{n}(x)$ and $\operatorname{Epi}\left(C_{\tau}^{\infty}\right)=\overrightarrow{\operatorname{Viab}}_{\Gamma_{\rho, \tau}}\left(\mathcal{H}_{\tau}^{h}\right)$ where $\Gamma_{\rho, \tau}(x, y):=\left\{(x, y)+\rho \Phi_{\rho}(x, y)\right\} \cap\left(X_{\tau} \times \tau \mathbb{Z}\right)$.

Let $\tau \rightarrow 0$. Thanks to the Refinement Principle [16] we have:

Proposition 3 The sequence $C_{\tau}^{\infty}(\cdot)$ converges to $C_{K^{s}, F}^{K^{h}}(\cdot)$ in the epigraphic sense: $\mathcal{E} p i\left(C_{K^{s}, F}^{K^{h}}\right)=\lim _{\tau \rightarrow 0} \mathcal{E} p i\left(C_{\tau}^{\infty}\right)$. Moreover $\forall x \in X_{\tau}, C_{\tau}^{\infty}(x) \leq C_{K^{s}, F}^{K^{h}}(x)$ and $C_{\tau}^{\infty}$ converges pointwisely to $C_{K^{s}, F}^{K^{h}}$

$$
\forall x \in K, C_{K^{s}, F}^{K^{h}}(x)=\lim _{p \rightarrow+\infty} \min _{x \in(x+\tau \mathcal{B}) \cap X_{\tau}} C_{\tau}^{\infty}(x)
$$


Epigraphical convergence follows from the Convergence Theorem of discrete viability kernels to the viability kernel and pointwise convergence follows from that viability kernels are epigraphs of l.s.c. functions $C_{K^{s}, F}^{K^{h}}$ and $C_{\tau}^{\infty}$.

\subsection{Example: the Goodwin employment-wages model}

We illustrate this approach by considering a production function $Y=f(K, L, t)=$ $f(K, L, t)=\min \left[\frac{K}{k}, L e^{q t}\right]$ depending on the capital $K$, the firm's ask for labor $L$ and the time $t$ where $\frac{K}{k}$ is the return of the capital and $L e^{q t}$ is the amount of working time necessary for producing $Y$. The growth rate of mean productivity for each worker $q$ is assumed to be constant (see [12, Goodwin]).

Production is shared between wages $w$ and benefits $\pi: Y=w L+\pi$. Benefits $\pi=I$ are fully reinvested and wages $w L=C$ are fully consummated so that at each time $Y=C+I$. Saving $S=r K$ is reinvested and increases capital $K$ : $\frac{d K}{d t}=r K$. Ask for labor $N$ increases with constant rate, following a demographic evolution of malthusian type : $N=N_{o} e^{n t}$.

Let be $x=\frac{L}{N}$ the employment rate. Phillips curve expresses the dependance of wages on the employment rate: $\frac{w^{\prime}}{w}=\beta x+(\alpha-\beta), \quad \beta>0$ and $y=\frac{w L}{Y}=\frac{C}{Y}$ denotes the part of the production intended for wages.

Assuming $\pi=I=S$, we have $\frac{r K}{Y}=1-y$ and $r=\frac{Y}{K}(1-y) \geq \frac{1}{k}(1-y)$.

Let us introduce an anticipative inflation/deflation control $\rho_{a} \in\left[\rho_{\min } ; \rho_{\max }\right]$ allowing Public Authority to intervene. The Goodwin model reads:

$$
\begin{aligned}
& x^{\prime}=x\left[\frac{1}{k}(1-y)-q-n\right] \\
& y^{\prime} \in y[\beta(x-1)-q]+\alpha\left[\rho_{\min } ; \rho_{\max }\right]
\end{aligned}
$$

Constraint $x \in[0,8 ; 1,2]$ expresses reasonable unemployment or call to foreign manpower and $y \in[0 ; 0,5]$ limits the part of the production activity devoted to wages.

An evolution may leave the constraint set and remain out for some lapse of time. One want to define an alert process to help in choosing a good policy in order to recover "as well as possible" acceptable economical situation, to "prevent" or to "minimize" future crisis. An answer to this problem is given thanks to the computation of the Minimal Crisis Time function.

But this is not necessarily the best measure crisis as shown on figure 4.5. We can observe that the minimal time is reached for a trajectory which crosses a very high wages level area.

Also, introducing Alert levels in the frame of hybrid dynamical system, we can extend the Minimal Crisis Time approach and introduce qualitative criteria.

It consists in minimizing a crisis cost function in order to take into account as well crisis time as seriousness degree of crisis. It also allows to apply specific procedures depending on the position of the state $x$ in or out of $K_{s}$.

\subsection{Hybrid System Approach and Alert Management}

Let us consider a finite partition (alert levels) $\left\{K_{i}\right\}_{i=0, \ldots, p}$ of $X$ with $K_{0}=K^{s}$ and $K_{p}=\overline{X \backslash K^{h}}$ and an index variable $y \in I=\{0, \ldots, p\}$, a cost variable 

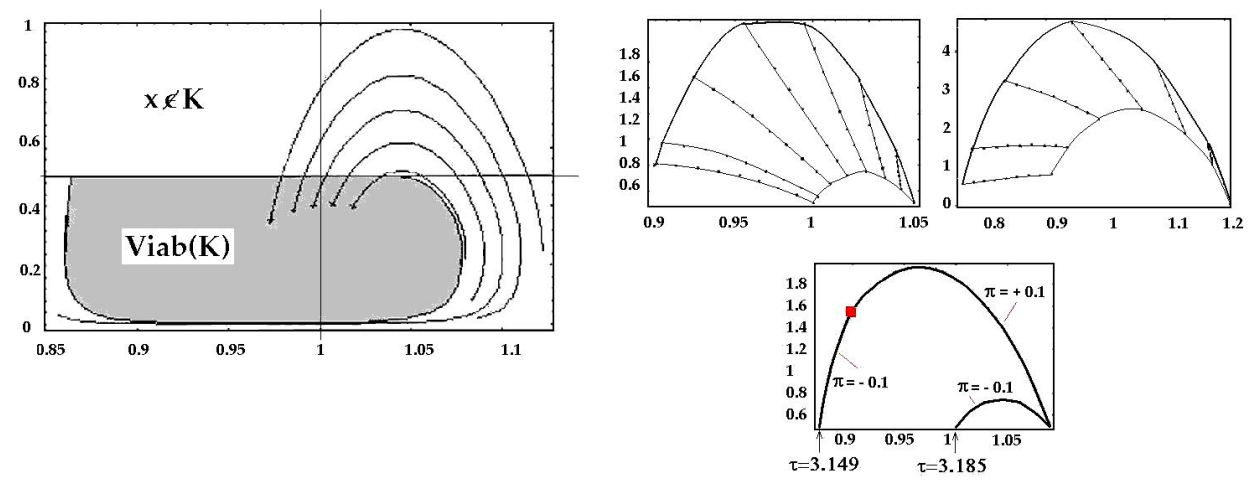

Fig. 1. Crisis Trajectories and isochronal curves. The Minimal time trajectory is obtained by taking $\pi=0.1$ in a first period and $\pi=-0.1$ in a second period.

$z \in R^{+}$and a transition set-valued map $\Phi: I \rightsquigarrow I$ defined on $\cup_{i \in I} \partial K_{i}$ by $\Phi(i)=\left\{j \in I \mid \partial K_{j} \cap \partial K_{i} \neq \emptyset\right\}$.

Consider the hybrid system:

Continuous Dynamic: $\left\{\begin{array}{l}x^{\prime}=f(x, y, u) \\ y^{\prime}=0 \\ z^{\prime}=-\gamma(y)\end{array} \quad ; \begin{array}{c}\text { Discrete Dynamic: } \\ X^{n+1} \in \Phi\left(X^{n}\right)\end{array}\left\{\begin{array}{l}x^{n+1}=x^{n} \\ y^{n+1} \in \phi\left(y^{n}\right) \\ z^{n+1}=z^{n}\end{array}\right.\right.$

where the constraint set $K:=\left\{(x, y, z) \in X \times I \times R, x \in K_{y}, z \geq 0\right\}$ and the reset set $C:=\left\{(x, y, z) \in X \times I \times R, x \in \cup_{i \in I} \partial K_{i}\right\}$.

Equation $y^{\prime}=0$ means that index $y$ does not change whenever the trajectory does not reach $\partial K_{y}$.

The function $\gamma(y)$ represents a "cost" (or penalties, weight,...) generated by the membership of $x$ to the alert level $K_{y}$ with $\gamma(0)=0$ and $\gamma(n)=+\infty$.

The variable $z(t)$ measures the total cost of the lapse from constraint $K_{0}$ from time 0 to $t$ associated with the partition and the cost function $\gamma(\cdot)$.

Conclusion - Thanks to the extension of viability techniques to hybrid calculus we can evaluate for any initial position how serious will be a present or future crisis taking into account the predetermined scale of seriousness and approximate the optimal control which corresponds to the Minimal Cost trajectory.

Since constraint and reset sets are compact, $F:(x, y, z) \rightsquigarrow f(x, y, u) \times\{0\} \times$ $-\gamma(y)$ is Marchaud and $(x, y, z) \rightsquigarrow x \times \Phi(y) \times z$ is upper semi-continuous with compact values, we deduce from the existence and convergence Theorems $[2$, Aubin], [17, Saint-Pierrre] that the Hybrid Kernel exists, is closed and that it can be approximated by a sequence of discrete viability kernels.

Also, $\left(x_{0}, y_{0}, z_{0}\right) \in H y b_{(F, \Phi)}(K)$ if and only if there exists an evolution starting from $x_{0}$ which belongs to the region $K_{y_{0}}$ and which will recover complete viability in finite time with a crisis cost at most equal to $z_{0}$.

Since the cost function is positive and since $K_{0}$ is compact, compacity of the constraint set is not required and, as for characterizing the Minimal Time 
function with constraints, we prove that the Hybrid Kernel exists and is the epigraph of the lower semicontinuous Minimal Crisis Cost function:

$$
\Gamma(x):=\inf \left\{z \operatorname{such} \text { that }(x, y, z) \in H y b_{F, \Phi}(K), y \in i(x)\right\} .
$$

Remark - The minimal Crisis Time function is a particular case where $p=2$, $\overline{K_{0}}=K^{s}, K_{1}=\overline{K^{h} \backslash K^{s}}, K_{2}=\overline{X \backslash K^{h}}, \gamma(0)=0, \gamma(1)=1$ and $\gamma(2)=+\infty$.

\section{References}

1. AUBIN J.-P. (1991) Viability Theory Birkhäuser, Boston, Basel, Berlin

2. AUBIN J.-P. (1999) Impulse Differential Inclusions and Hybrid Systems: A Viability Approach, Lecture Notes, University of California at Berkeley

3. AUBIN J.-P. \& FRANKOWSKA H. (1990) Set-Valued Analysis,

4. BAYEN A., CRÜCK E. \& TOMLIN C., Guaranteed overapproximations of unsafe sets for continuous and hybrid systems: solving the Hamilton-Jacobi equation using viability techniques, LNCS, V. 2289, Springer. (2002)

5. BENSOUSSAN A. \& MENALDI (1997) Hybrid Control and Dynamic Programming, Dynamics of Continuous, Discrete and Impulse Syst.,3,395-442

6. CARDALIAGUET P., QUINCAMPOIX M. \& SAINT-PIERRE P. (1999) Set-valued numerical methods for optimal control and differential games, In Stochastic And Differential Games. TheOry AND Numerical MethODS, Annals of the Intern. Soc. of Dynamical Games, 177-247 Birkhäuser

7. CRÜCK E. (2001) Problèmes de cible sous contrainte d'état pour des systèmes non linéaires avec sauts d'état, C.R.A.S. Paris, t.333 Série I p.403-408

8. CRÜCK E. (2002) Thèse en préparation.

9. DORDAN O. (1995) Analyse qualitative, Masson

10. DOYEN L. \& SAINT-PIERRE P. (1997) Scale of viability and minimal time of crisis, Set-Valued Analysis, 5, 227-246

11. FRANKOWSKA H. (1991) Lower semicontinuous solutions to HamiltonJacobi-Bellman equations, Proc. of 30th CDC Conference, IEEE, Brighton.

12. GOODWIN R.M. (1967) A Growth Cycle, in Feinstein, editor, Socialism, Capitalism and Economic Growth.

13. LEITMANN G., (1979) Guaranteed Asymptotic Stability for a Class of Uncertain Linear Dynamical Systems. J. of Optim. Theory \& Appl., 27(1).

14. QUINCAMPOIX M. (1992) Differential inclusions and target problems, SIAM J. Control and Optimization, 30, 324-335

15. QUINCAMPOIX M. \& VELIOV V. (1998) Viability with a target: theory and applications, in Applic. of math. in engineering, 47-54, Heron Press

16. SAINT-PIERRE P., (1994) Approximation of the Viability Kernel, Applied Mathematics \& Optimization, 29 (1994), 187-209.

17. SAINT-PIERRE P., (2002) Hybrid Kernels and Capture Basins for Impulse Constrained Systems, Proceedings of Hybrid Systems: Computation and Control, Lecture Notes in Computer Science, 2289, Springer

18. SEUBE N. (1995) Robust Stabilization of Uncertain Systems. Journal of Math. Analysis and Applications, 452-466.

19. SASTRY S., (1999) Non Linear Systems. Analysis, Stability And Control, Springer-Verlag. 